\title{
Alternatives to Traditional EMS Dispatch and Transport: A Scoping Review of Reported Outcomes
}

\author{
Jan L. Jensen, MAHSR ${ }^{{ }^{\dagger}}$; Alix J.E. Carter, MD, MPH*; Jennifer Rose*; Sarah Visintini, BA, MLIS ${ }^{\ddagger}$; \\ Emmanuelle Bourdon, MSHSA, BA ${ }^{\S \pi}$; Ryan Brown, BSc*; Jennifer McVey, MD, MSc* ${ }^{*}$; Andrew $\mathrm{H}^{*}$ \\ Travers, MD, MSc ${ }^{*}$
}

\section{ABSTRACT}

Objectives: Emergency medical services (EMS) programs, which provide an alternative to traditional EMS dispatch or transport to the emergency department (ED), are becoming widely implemented. This scoping review identified and catalogued all outcomes used to measure such alternative EMS programs.

Data Source: Broad systematized bibliographic and grey literature searches were conducted.

Study Selection: Inclusion criteria were 911 callers/EMS patients, reported on alternatives to traditional EMS dispatch OR traditional EMS transport to the ED, and reported an outcome measure.

Data Extraction: The reports were categorized as either alternative to dispatch or to EMS transport, and outcome measures were categorized and described.

Data Synthesis: The bibliographic search retrieved 13,215 records, of which 34 articles met the inclusion criteria, with an additional 10 added from reference list hand-searching ( $n=44$ included). In the grey literature search, 31 websites were identified, from which four met criteria and were retrieved ( $n=4$ included). Fifteen reports (16 studies) described alternatives to EMS dispatch, and 33 reports described alternatives to EMS transport. The most common outcomes reported in the alternatives to EMS dispatch reports were service utilization and decision accuracy. Twenty-four different specific outcomes were reported. The most common outcomes reported in the alternatives to EMS transport reports were service utilization and safety, and 50 different specific outcomes were reported.

Conclusions: Numerous outcome measures were identified in reports of alternative EMS programs, which were catalogued and described. Researchers and program leaders should achieve consensus on uniform outcome measures, to allow benchmarking and improve comparison across programs.

\section{RÉSUMÉ}

Objectif: Les programmes de services médicaux d'urgence (SMU), qui offrent une solution de rechange à la répartition traditionnelle des SMU ou au transport classique des malades aux services des urgences (SU), gagnent de plus en plus de terrain. Dans l'examen d'établissement de la portée décrit ici, les auteurs ont relevé et classé tous les résultats utilisés pour mesurer l'efficacité de ces programmes novateurs de SMU.

Sources des données: Les auteurs ont entrepris une vaste recherche systématisée de références bibliographiques, et effectué des recherches méthodiques dans la documentation parallèle.

Sélection des études: Les critères de sélection comprenaient les appels faits aux services d'urgence 911 ou les malades examinés par les SMU, inscrits dans les rapports de solutions de rechange à la répartition traditionnelle des SMU OU au transport classique des malades au SU par les SMU, ainsi que l'existence d'un critère d'évaluation.

Extraction des données: Les rapports ont été classés soit dans la catégorie des solutions de rechange à la répartition des services, soit dans la catégorie des solutions de rechange aux transports effectués par les SMU; quant aux critères d'évaluation, ils ont été classés et décrits.

Synthèse des données: La recherche dans les références bibliographiques a permis de relever 13215 documents, dont 34 articles répondaient aux critères de sélection; à cela s'ajoutent 10 autres documents provenant d'une recherche manuelle dans des listes de références $(n=44$ documents sélectionnés). Quant à la recherche dans la documentation parallèle, elle a permis de relever 31 sites Web, dont 4 répondaient aux critères de sélection; ils ont tous été extraits ( $n=4$ sites Web sélectionnés). Dans 15 rapports (16 études), il y avait une description des solutions de rechange à la répartition des SMU, et, dans 33 rapports, une description des solutions de rechange au transport par les SMU. Les résultats indiqués le plus souvent dans les rapports de solutions de rechange à la répartition des SMU étaient I'utilisation des services et la justesse des décisions; se sont dégagés des documents 24 résultats différents. Les résultats indiqués le plus souvent dans les rapports de solutions de rechange au transport par les SMU étaient I'utilisation des services et la sécurité; se sont dégagés des documents 50 résultats différents.

From the *Nova Scotia Emergency Health Services, Halifax, NS; †Department of Emergency Medicine, Division of EMS, Dalhousie University, Halifax, NS; ‡Dalhousie University Libraries, Halifax, NS; §Collège Ahuntsic, Montreal, QC; and ๆUniversité de Québec à Montréal, Montreal, QC.

Correspondence to: Jan L Jensen, 239 Brownlow Avenue, Suite 300, Dartmouth Nova Scotia B3B2B2; jan.jensen@emci.ca 
Conclusions: De nombreux critères d'évaluation ont été relevés dans les rapports de programmes de SMU novateurs, critères qui ont été classés et décrits. Les chercheurs et les responsables des programmes devraient établir un consensus sur des critères uniformes d'évaluation afin de permettre
I'établissement de normes de référence et d'améliorer les comparaisons entre programmes.

Keywords: emergency medical services, dispatch, transport, alternatives, scoping review

\section{INTRODUCTION}

In recent years, there has been an increase in emergency medical services (EMS) services that are alternatives to EMS dispatch or EMS transport to the emergency department (ED). Traditionally, ambulances are dispatched for all 911 callers. All patients are transported to the ED, unless the patient or decision maker refuses transport. A "multiple option decision point" has been previously described as an alternative, in which a decision on the need for EMS transport could be made at two points: at the EMS dispatch centre and on scene, ${ }^{1,2}$ in an attempt to "get the right patient to the right place at the right time" (Figure 1).

Emergency medical dispatching conceptually consists of two major tasks: call processing and dispatching of EMS resources. Call processing can be further broken down into: 1) triage (deciding whether to send emergency resources or not); 2) prioritization (how quickly to send resources; and 3) choosing the level of resources required. ${ }^{3}$ Alternatives to EMS dispatch may be decided during the triage or choosing resources stages. On-scene alternatives to EMS transport to the ED include protocols to treat the patient and leave them on scene ("treat and release"), or to treat and refer to other parts of the healthcare system. These types of alternatives have been included in the expanding

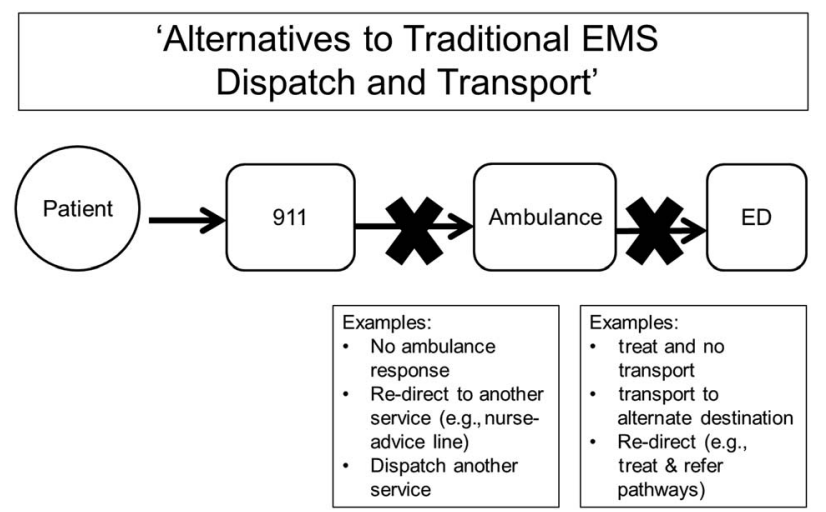

Figure 1. Alternatives to Traditional EMS Dispatch and EMS Transport to the ED. scope of "community paramedicine," also more recently described as "mobile integrated healthcare" and "patient-centered EMS."

A narrative literature review of on-scene alternatives to ED transport conducted by Snooks and colleagues 10 years ago found that there were few comparative studies and that data were too scarce to determine the safety of such programs. ${ }^{5}$ More recently, a community paramedicine systematic review drew similar findings. ${ }^{6}$ This variance in the interventions studied, methods used, and outcomes measured led to a body of knowledge that is difficult to synthesize and interpret, and nearly impossible to generalize to local settings.

To aid future researchers and those measuring quality and safety in such alternative EMS programs, this broad scoping review sought to identify, catalogue, and describe the outcome measures reported by such programs.

\section{METHODS}

\section{Study design}

This scoping review was based on Arksey and O'Malley's methodological framework, ${ }^{7}$ and consisted of three searching techniques: 1) a systematic search of bibliographic databases for research literature on the topic; 2) a hand search of websites for grey literature; and 3) a snowball search of the reference lists of articles that met the inclusion criteria. The bibliographic and grey literature searches were purposefully broad in order to capture as much of the research conducted in this field as possible.

\section{Data source}

The bibliographic database search was developed using a pearl growing search strategy, in which key articles identified by the investigators were mined for index terms and keywords. ${ }^{8-11}$ Seven articles were used for this purpose. ${ }^{12-18}$ The database search was conducted in 
PubMed, EMBASE, CINAHL and the Cochrane Library in 2012 and repeated on May 19, 2014. The search was created using a combination of Medical Subject Headings (MeSH) and keywords in PubMed, and mapped to the other databases using their designated thesauri. The three main concepts of interest in this search were: 1) 911 callers/EMS patients; 2) interventions and services provided by EMS; and 3) decision making. These were combined with "AND," and "OR" (Appendix A). English and human subject limiters were applied.

The grey literature search was conducted from November 4, 2012 to December 4, 2012 by two investigators and a paramedic, who hand-searched a list of websites (Appendix B). This list was generated from suggestions made by investigators, as well as an exploratory search of government and health association websites for possible sites of interest. ${ }^{19,20}$ The searches and findings were documented, along with any links followed. The snowball search was conducted by reviewing the titles of articles in the reference lists of included articles.

\section{Study selection}

For a record to be included, it had to contain each of the following: a) the population was 911 callers or EMS patients, b) it described an alternative to traditional EMS dispatch OR to transport to the $\mathrm{ED}$, and c) it reported an outcome measure. Abstracts were excluded. Reviews were not included, although reference lists were hand-searched for primary reports.

Review for inclusion of the bibliographic database search was first conducted by title by a single author. Review for inclusion by abstract, full text article, and retrieved grey literature was conducted by two independent authors with a third author serving as adjudicator.

\section{Data extraction}

The intervention described in each report was categorized as either an alternative to EMS dispatch or alternative to EMS transport to ED. Each outcome reported in the included reports was categorized into one of the following categories, which were determined a priori by study team knowledge of the literature and consensus: 1) clinical, 2) safety, 3) service utilization, 4) patient satisfaction, 5) cost, 6) accuracy of decision,

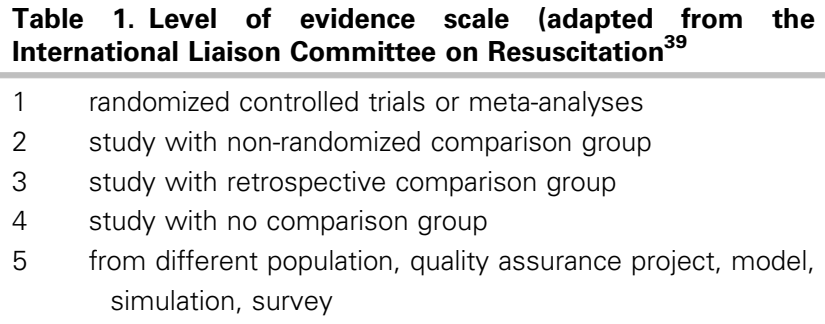

7) process outcome, and 8) other. The included reports were divided among team members for abstraction. Study team members determined the Level of Evidence (Table 1), Direction of Evidence and outcome category for each study through discussion and consensus. The Direction of Evidence for each study was based on the results of the primary outcome (adapted from the Canadian Prehospital Evidence-based Practice Project). ${ }^{40}$

\section{RESULTS}

In total, 13,215 records were retrieved through the bibliographic database searches, including the seven pearl articles. These were imported into the reference management citation software Refworks (Proquest, Bethesda, MD, USA); 2,598 duplicates were then eliminated, leaving 10,617 unique records. Thirty-seven websites were hand-searched for grey literature, in which 31 records of interest were identified. A total of 48 records met the inclusion criteria: 34 from the bibliographic search, four from the grey literature search, and 10 from the snowball search (Figure 2).

\section{Outcome measures reported on alternatives to EMS dispatch}

Fifteen reports were categorized as alternatives to EMS dispatch (Table 2). One report described two separate studies. ${ }^{25}$ Articles were from the UK $(n=6)$, the United States $(n=6)$, Canada $(n=2)$, and $\operatorname{Iran}(n=1)$. Eleven of these EMS systems were paramedicbased, ${ }^{12,13,15,16,18,21-26}$ two were physician-based with basic emergency medical technicians, ${ }^{3,27}$ and in one report the type of EMS system was unclear. ${ }^{28}$ The following interventions were studied: transferring 911 callers to nurse-advice lines $(n=6)$, EMS dispatch providing advice or self-care instructions $(n=4)$, alternative EMS response being dispatched $(n=3)$, and 


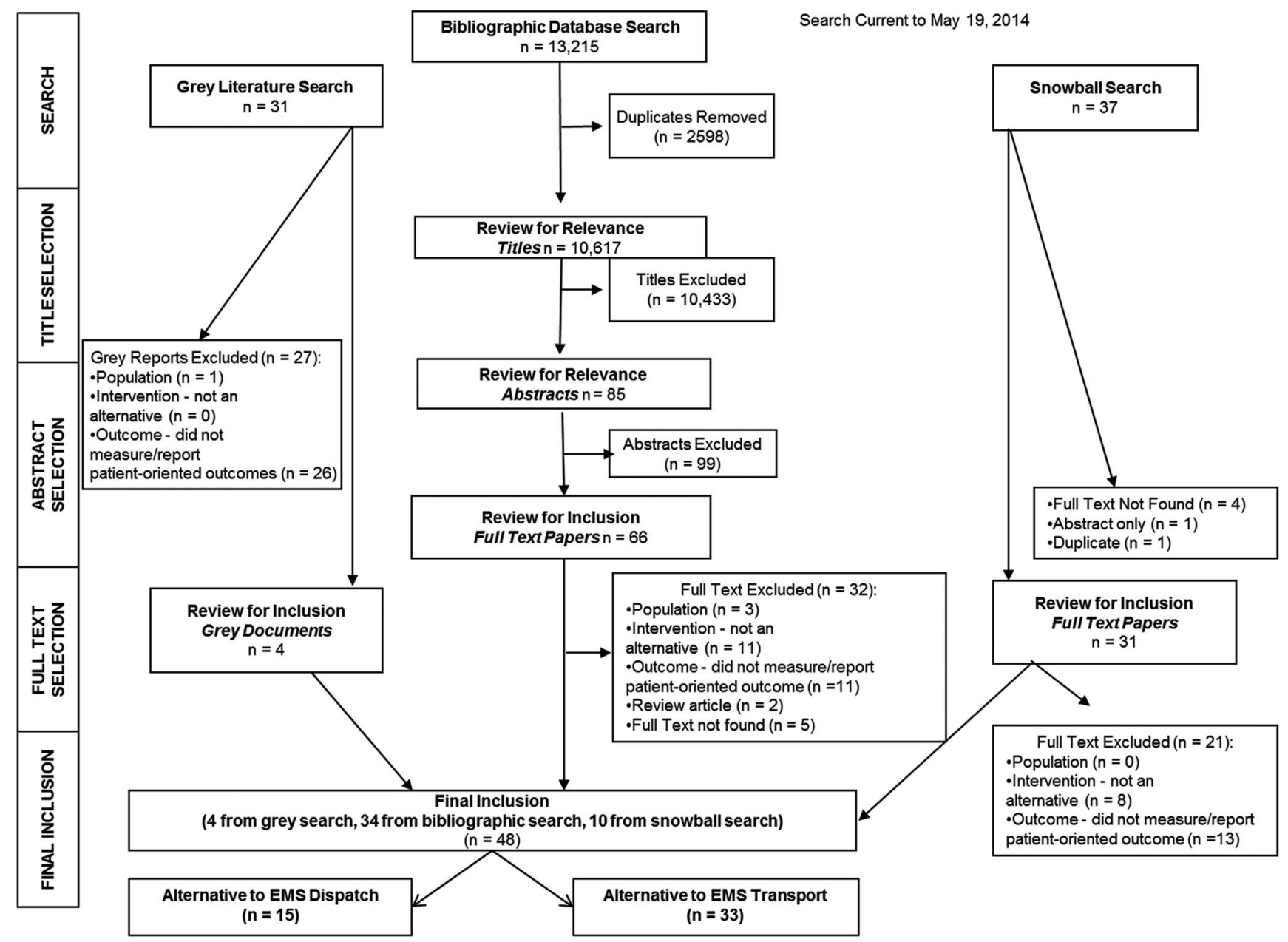

Figure 2. Scoping review flow diagram.

identification of low acuity calls that do not require EMS response $(n=2)$. Studies were most commonly those without a comparison group (6/16), and studies with alternative designs, such as patient surveys or models (4/16). There were two uncontrolled designs with a comparison group, three studies with a retrospective comparison group and one controlled trial. The results of five studies were considered supportive of the intervention, 10 studies had neutral results and one study had opposing results. The outcome categories, reported by the greatest number of reports, were service utilization $(n=12)$ and decision accuracy $(n=7)$ (Table 2). In total, there were 24 unique outcomes reported. The outcome categories with the greatest number of specific different outcomes were service utilization $(n=8)$, accuracy of decision $(n=4)$, safety $(n=3)$, call times $(n=3)$, cost $(n=3)$, patient satisfaction $(n=2)$, and process outcomes $(n=1)$.

\section{Outcome measures reported on alternatives to EMS transport}

Thirty-three reports were categorized as alternatives to EMS transport to the ED, all of which were from paramedic-based systems (Table 3). Sixteen studies were from the UK, 13 were from the US, two from Australia, one from Sweden and one from Canada. Twelve reported on expanded-scope EMS programs (such as the UK emergency care practitioner), 10 reported on EMS-initiated non-transport, six described programs in which calls were referred to another health service, three were of treat-and-release protocols, one assessed using telemedicine to expand consults by EMS for non-transport, and one studied providing patients with an alternative mode of transport to the ED (via taxi). The majority of study designs were those without a comparison group $(n=14)$, followed by studies with a retrospective comparison group $(n=6)$. There were 


\begin{tabular}{|c|c|c|c|c|c|c|c|c|}
\hline First Author & $\begin{array}{c}\text { Year of } \\
\text { Publication }\end{array}$ & Country & Service Studied & $\mathrm{n}$ & $\begin{array}{l}\text { Level of } \\
\text { Evidence of } \\
\text { Study Design }\end{array}$ & $\begin{array}{l}\text { Direction of } \\
\text { Evidence of } \\
\text { Primary Outcome }\end{array}$ & Outcome Category & Specific Outcome Reported \\
\hline $\begin{array}{l}\text { Research and } \\
\text { Quality }^{21}\end{array}$ & & & & & & & Cost & $\begin{array}{l}\text { Annual EMS transport cost } \\
\text { estimates }\end{array}$ \\
\hline Alizadeh ${ }^{28}$ & 2012 & Iran & $\begin{array}{l}\text { Identify low-acuity } \\
\text { calls that did not } \\
\text { require ambulance } \\
\text { response }\end{array}$ & 200 & 3 & Supportive & Service Utilization & Unnecessary missions \\
\hline Crowther $^{22}$ & 2009 & UK & $\begin{array}{l}911 \text { callers to } \\
\text { nurse-advice line }\end{array}$ & 3041 & 4 & Supportive & Service Utilization & 911 calls directed to alternative \\
\hline \multirow[t]{2}{*}{ Dale $^{13}$} & 2004 & UK & $\begin{array}{l}\text { Provided advice or } \\
\text { self-care }\end{array}$ & 239 & 5 & Supportive & Safety & $\begin{array}{l}\text { Panel assessment of whether } \\
\text { decision was safe }\end{array}$ \\
\hline & & & instructions & & & & Accuracy of Decision & $\begin{array}{l}\text { Triage decision compared to } \\
\text { expert panel }\end{array}$ \\
\hline Studnek ${ }^{18}$ & 2012 & USA & $\begin{array}{l}911 \text { callers to } \\
\text { nurse-advice line }\end{array}$ & $\begin{array}{l}1078 \text { intervention, } \\
1862 \text { control }\end{array}$ & 4 & Neutral & Accuracy of Decision & $\begin{array}{l}\text { Triage decision compared to } \\
\text { subsequent ED attendance or } \\
\text { hospital admission }\end{array}$ \\
\hline Widiatmoko ${ }^{41}$ & 2008 & UK & $\begin{array}{l}911 \text { call to alternative } \\
\text { EMS response }\end{array}$ & $\begin{array}{l}198 \text { intervention, } \\
2583 \text { control }\end{array}$ & 3 & Neutral & Cost & $\begin{array}{l}\text { Incremental cost-effectiveness } \\
\text { analysis }\end{array}$ \\
\hline \multirow[t]{5}{*}{ Turner $^{25}(a)^{*}$} & 2006 & UK & $\begin{array}{l}911 \text { callers to } \\
\text { nurse-advice line }\end{array}$ & $\begin{array}{l}1766 \text { intervention, } \\
2158 \text { control }\end{array}$ & 1 & Neutral & Service Utilization & $\begin{array}{l}\text { Calls returned from nurse advice } \\
\text { line back to EMS dispatch }\end{array}$ \\
\hline & & & & & & & Time & $\begin{array}{l}\text { EMS response intervals, total } \\
\text { episode time, EMS job cycle } \\
\text { time }\end{array}$ \\
\hline & & & & & & & Patient Satisfaction & $\begin{array}{l}\text { Patient satisfaction and } \\
\text { acceptability of new service }\end{array}$ \\
\hline & & & & & & & Accuracy of Decision & $\begin{array}{l}\text { Final disposition of nurse } \\
\text { assessment }\end{array}$ \\
\hline & & & & & & & Cost & Economic analysis modelling \\
\hline
\end{tabular}




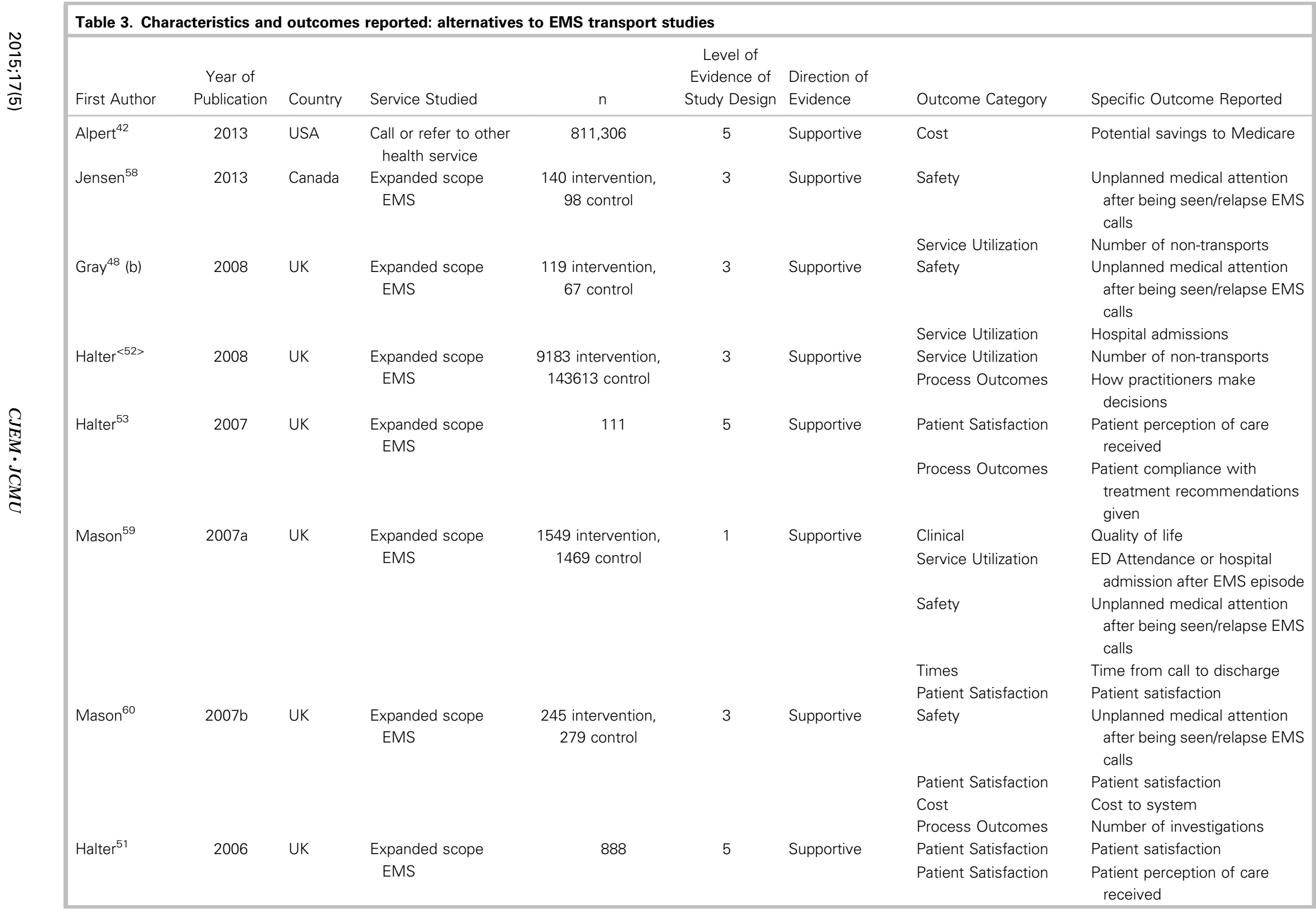




\begin{tabular}{|c|c|c|c|c|c|c|c|c|c|}
\hline & Table 3. (Cc & tinued ) & & & & & & & \\
\hline \multirow{15}{*}{ 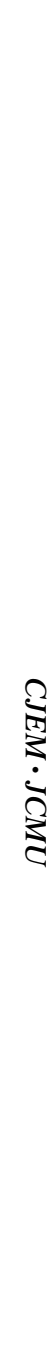 } & First Author & $\begin{array}{c}\text { Year of } \\
\text { Publication }\end{array}$ & Country & Service Studied & $\mathrm{n}$ & $\begin{array}{l}\text { Level of } \\
\text { Evidence of } \\
\text { Study Design }\end{array}$ & $\begin{array}{l}\text { Direction of } \\
\text { Evidence }\end{array}$ & Outcome Category & Specific Outcome Reported \\
\hline & Snooks ${ }^{17}$ & $2004 a$ & UK & $\begin{array}{l}\text { Treat and Release } \\
\text { (includes } \\
\text { assessment tools }\end{array}$ & $\begin{array}{l}409 \text { intervention, } \\
425 \text { control }\end{array}$ & 1 & Supportive & Safety & $\begin{array}{l}\text { Unplanned medical attention } \\
\text { after being seen/relapse EMS } \\
\text { calls }\end{array}$ \\
\hline & & & & to leave patients) & & & & Service Utilization & Number of non-transports \\
\hline & & & & & & & & Times & EMS job cycle time \\
\hline & & & & & & & & Patient Satisfaction & Patient satisfaction \\
\hline & Cooper ${ }^{45}$ & 2004 & UK & Expanded scope & 170 intervention, & 2 & Supportive & Service Utilization & Number of non-transports \\
\hline & & & & EMS & 331 control & & & $\begin{array}{l}\text { Clinical } \\
\text { Process Outcomes }\end{array}$ & $\begin{array}{l}\text { Diagnosis/chief complaint } \\
\text { Paramedic documentation }\end{array}$ \\
\hline & Lerner $^{57}$ & 2003 & USA & $\begin{array}{l}\text { Treat and Release } \\
\text { (includes } \\
\text { assessment tools } \\
\text { to leave patients) }\end{array}$ & 36 & 4 & Supportive & $\begin{array}{l}\text { Safety } \\
\text { Patient Satisfaction } \\
\text { Process Outcomes }\end{array}$ & $\begin{array}{l}\text { Complications after call } \\
\text { Patient satisfaction } \\
\text { Paramedic compliance with } \\
\text { protocols }\end{array}$ \\
\hline & Schaefer ${ }^{14}$ & 2002 & USA & $\begin{array}{l}\text { Call or refer to other } \\
\text { health service }\end{array}$ & $\begin{array}{l}1016 \text { intervention, } \\
2617 \text { control }\end{array}$ & 3 & Supportive & $\begin{array}{l}\text { Safety } \\
\text { Service Utilization }\end{array}$ & $\begin{array}{l}\text { Case review } \\
\text { ED Attendance after EMS } \\
\text { episode }\end{array}$ \\
\hline & & & & & & & & Service Utilization & Number of clinical referrals \\
\hline & & & & & & & & Service Utilization & Number of non-transports \\
\hline & & & & & & & & Accuracy of Decision & $\begin{array}{l}\text { Appropriate decision for } \\
\text { alternative to ED care }\end{array}$ \\
\hline & Coates $^{44}$ & 2012 & UK & $\begin{array}{l}\text { Expanded scope } \\
\text { EMS }\end{array}$ & 172 & 4 & Neutral & Accuracy of Decision & $\begin{array}{l}\text { Whether decision resulted in } \\
\text { avoided ED visit or hospital } \\
\text { admission }\end{array}$ \\
\hline & Dixon $^{47}$ & 2009 & UK & $\begin{array}{l}\text { Expanded scope } \\
\text { EMS }\end{array}$ & $\begin{array}{c}1446 \text { intervention, } \\
1408 \text { control }\end{array}$ & 1 & Neutral & Cost & Economic evaluation \\
\hline & Knapp ${ }^{56}$ & 2009 & USA & $\begin{array}{l}\text { Alternative mode of } \\
\text { transport to ED }\end{array}$ & 93 & 4 & Neutral & $\begin{array}{l}\text { Clinical } \\
\text { Times }\end{array}$ & $\begin{array}{l}\text { Hospital admission } \\
\text { Diagnosis/chief complaint } \\
\text { Time to ED triage }\end{array}$ \\
\hline $\begin{array}{l}\tilde{O} \\
\text { ज़ } \\
\stackrel{\vec{\Delta}}{\text { जv }}\end{array}$ & Cooper $^{46}$ & 2008 & UK & $\begin{array}{l}\text { Expanded scope } \\
\text { EMS }\end{array}$ & 611 & 4 & Neutral & $\begin{array}{l}\text { Clinical } \\
\text { Times } \\
\text { Service Utilization } \\
\text { Accuracy of Decision }\end{array}$ & $\begin{array}{l}\text { Diagnosis/chief complaint } \\
\text { EMS time on scene } \\
\text { Number of non-transports } \\
\text { Between dispatch, ECP and } \\
24 \text { hour hospital diagnosis }\end{array}$ \\
\hline
\end{tabular}




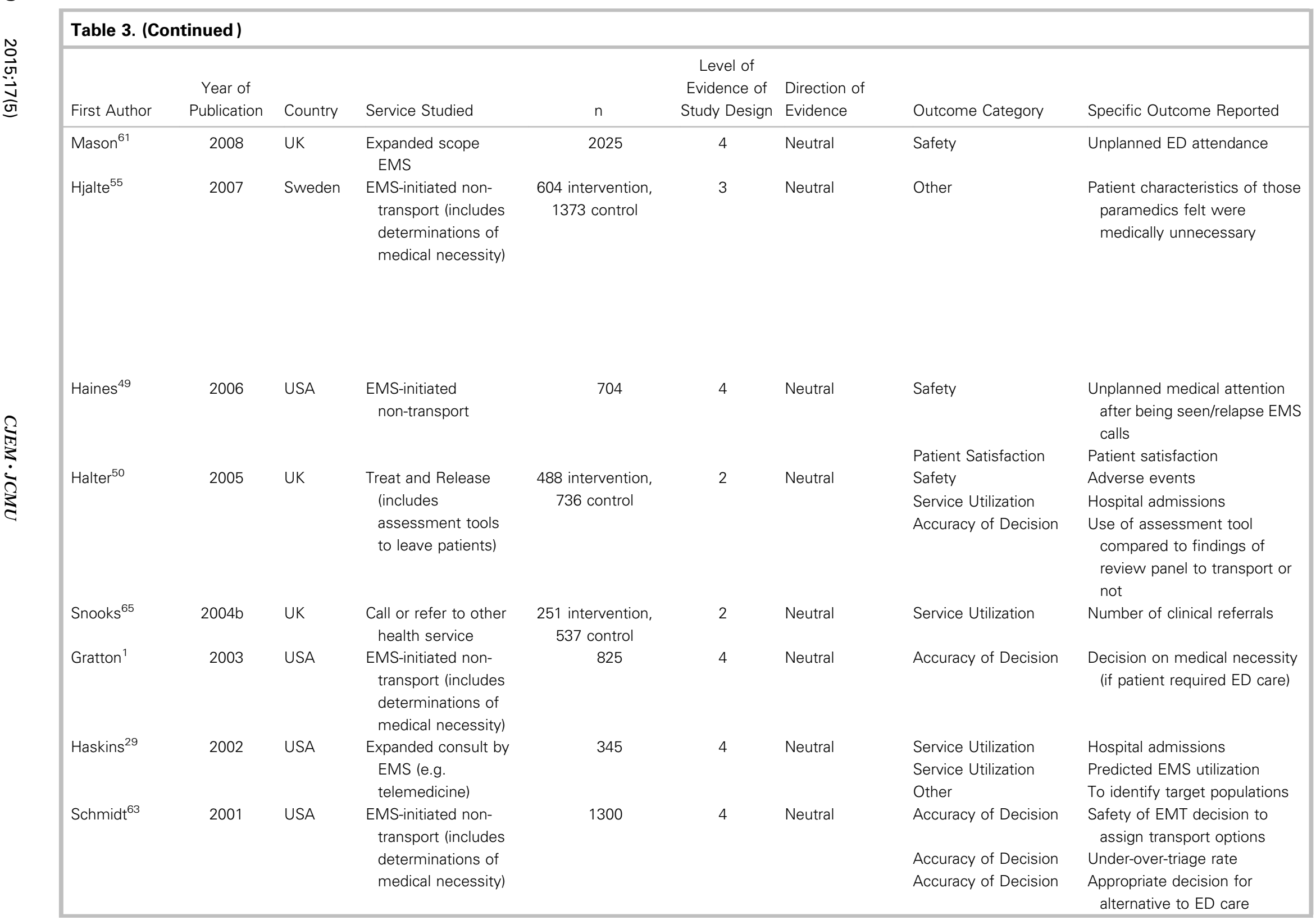




\begin{tabular}{|c|c|c|c|c|c|c|c|c|c|}
\hline & Table 3. (Co & tinued ) & & & & & & & \\
\hline \multirow{12}{*}{ 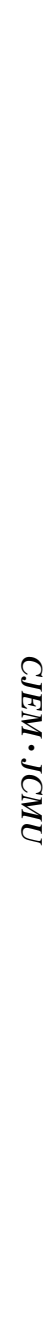 } & First Author & $\begin{array}{l}\text { Year of } \\
\text { Publication }\end{array}$ & Country & Service Studied & $\mathrm{n}$ & $\begin{array}{l}\text { Level of } \\
\text { Evidence of } \\
\text { Study Design }\end{array}$ & $\begin{array}{l}\text { Direction of } \\
\text { Evidence }\end{array}$ & Outcome Category & Specific Outcome Reported \\
\hline & Hauswald ${ }^{54}$ & 2002 & USA & $\begin{array}{l}\text { EMS-initiated non- } \\
\text { transport (includes } \\
\text { determinations of } \\
\text { medical necessity) }\end{array}$ & 183 & 4 & Opposing & Accuracy of Decision & $\begin{array}{l}\text { Decision on medical necessity } \\
\text { (if patient required ED care) }\end{array}$ \\
\hline & Silvestri $i^{64}$ & 2002 & USA & $\begin{array}{l}\text { EMS-initiated non- } \\
\text { transport (includes } \\
\text { determinations of } \\
\text { medical necessity) }\end{array}$ & 313 & 4 & Opposing & Accuracy of Decision & $\begin{array}{l}\text { Decision on medical necessity } \\
\text { (if patient required ED care) } \\
\text { documented by paramedics } \\
\text { compared to care actually } \\
\text { received in ED }\end{array}$ \\
\hline & Kamper $^{30}$ & 2001 & USA & $\begin{array}{l}\text { EMS-initiated non- } \\
\text { transport (includes } \\
\text { determinations of } \\
\text { medical necessity) }\end{array}$ & 1103 & 4 & Opposing & Other & To identify target populations \\
\hline & Pointer ${ }^{62}$ & 2001 & USA & $\begin{array}{l}\text { EMS-initiated non- } \\
\text { transport (includes }\end{array}$ & 1180 & 4 & Opposing & Accuracy of Decision & $\begin{array}{l}\text { Decision on medical necessity } \\
\text { (if patient required ED care) }\end{array}$ \\
\hline & & & & $\begin{array}{l}\text { determinations of } \\
\text { medical necessity) }\end{array}$ & & & & Accuracy of Decision & Under/over-triage rate \\
\hline & Zachariah $^{68}$ & 1992 & USA & $\begin{array}{l}\text { EMS-initiated } \\
\text { non-transport }\end{array}$ & 93 & 4 & Opposing & $\begin{array}{l}\text { Clinical } \\
\text { Safety }\end{array}$ & $\begin{array}{l}\text { Hospital admission } \\
\text { Unplanned medical attention } \\
\text { after being seen/relapse EMS } \\
\text { calls }\end{array}$ \\
\hline & & & & & & & & Patient Satisfaction & Patient satisfaction \\
\hline & Mikolaizak $^{31}$ & 2013 & Australia & $\begin{array}{l}\text { Call or refer to other } \\
\text { health service }\end{array}$ & 0 & 1 (Protocol) & & $\begin{array}{l}\text { Clinical } \\
\text { Service Utilization }\end{array}$ & $\begin{array}{l}\text { Subsequent fall } \\
\text { Number of contacts with health } \\
\text { system }\end{array}$ \\
\hline & & & & & & & & Process Outcome & $\begin{array}{l}\text { Uptake of recommendations by } \\
\text { patient }\end{array}$ \\
\hline & Snooks $^{67}$ & 2012 & UK & $\begin{array}{l}\text { Call or refer to other } \\
\text { health service }\end{array}$ & 0 & 1 (Protocol) & & Service Utilization & $\begin{array}{l}\text { Effect on EMS system } \\
\text { performance measures }\end{array}$ \\
\hline & & & & & & & & Clinical & Deaths \\
\hline \multirow{5}{*}{$\begin{array}{l}\text { Oे } \\
\text { ज़े } \\
\stackrel{\vec{\Delta}}{v}\end{array}$} & & & & & & & & & Hospital admission \\
\hline & & & & & & & & & Subsequent fall \\
\hline & & & & & & & & & $\begin{array}{l}\text { Quality of life } \\
\text { Quality-adjusted life years }\end{array}$ \\
\hline & & & & & & & & Patient Satisfaction & Patient satisfaction \\
\hline & & & & & & & & Cost & Patient care costs \\
\hline
\end{tabular}




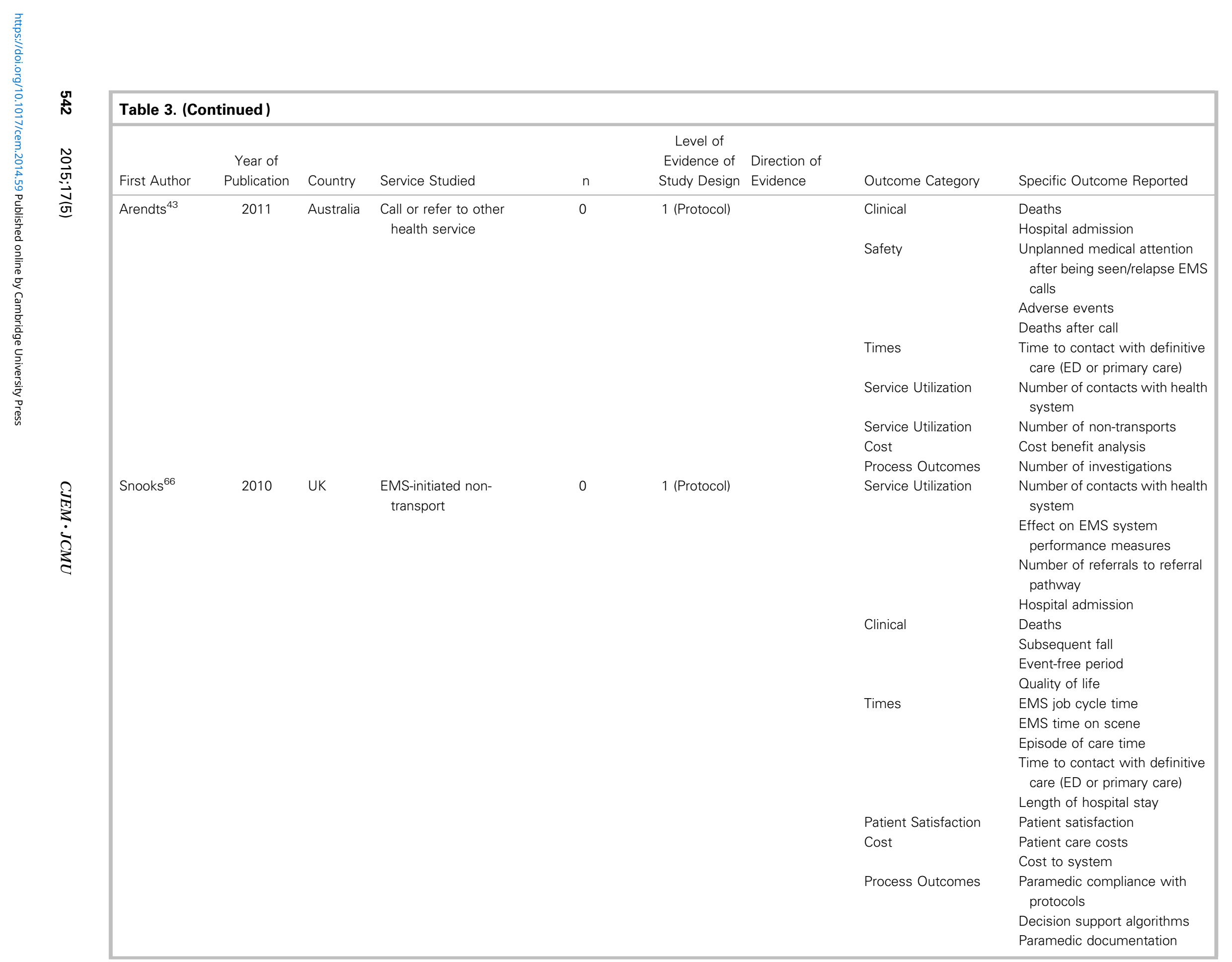


Table 4. Outcome categories, specific options and measurement considerations

Outcome Type

(Structure, Process,

System, Outcome)

Outcome Category

Specific Outcome Options

Measurement Considerations

Outcome

Clinical

- Deaths ${ }^{43,66,67}$

- Hospital admission $43,48,56,59,66-68$

- Subsequent fall ${ }^{31,66,67}$

- Event-free period ${ }^{66}$

- Quality of life 59,66,67

- Quality-adjusted life years ${ }^{67}$

- Diagnosis/chief complaint ${ }^{45,46,56}$

Outcome

Safety

- Deaths after call ${ }^{14,43}$

- Unplanned medical attention after being seen/relapse EMS calls ${ }^{17,43,48,49,58-60,68}$

- Complications after the call ${ }^{57}$

- Adverse events $16,25,43,50$

- EMS response intervals $s^{12,25}$

- EMS job cycle time ${ }^{17,24,25,66}$

- EMS time on scene $e^{46,66}$

- Episode of care time ${ }^{66}$

- Time to contact with definitive care (ED or primary (are) $)^{43,66}$

- Time to ED triage ${ }^{56}$

- Length of hospital stay ${ }^{66}$

- Time from call to discharge ${ }^{59}$

- Criteria should include consideration if cause of death was for a reason unrelated to EMS call.

- Define ideal length of time to follow patients, for example: 24 hours, $^{61} 48$ hours, $^{58} 28$ days $^{48}$.

- Options for data collection are to contact patient to determine outcome after non-transport ${ }^{49}$ or from clinical or administrative data (including EMS dispatch, EDs, hospital, family physician, other).

- Consensus needed for what is considered a "complication."

- Data may be collected from patients or chart review. ${ }^{16}$

- Consensus required on adverse event definitions, such as: A serious adverse event is "an unpredicted death or admission to hospital within two weeks of the original attendance." An adverse event is "an unpredicted use of the health service within two weeks of original attendance.". ${ }^{50}$

- Choose time points in which data entry for these fields by dispatch, EMS clinicians or hospital staff is mandatory, to minimize missing data.

- Consensus required on clear definitions for each time point and interval. Report each time point and interval clearly. 
Table 4. (Continued)

Outcome Type

(Structure, Process,

System, Outcomel

System Service Utilization $\quad 911$ calls directed to alternative 14,22,26,65,66 $^{\text {- }}$

Measurement Considerations

- Clearly define study patients, e.g., number of calls referred/total eligible calls. ${ }^{22,26}$

- Calls returned from alternative (e.g., nurse advice line) back to EMS dispatch ${ }^{16,25}$

- Number of EMS responses ${ }^{16,21}$

- Unnecessary missions ${ }^{28}$

- Predicted EMS utilization ${ }^{29}$

- Number of nontransports ${ }^{14,17,24,29,43,45,46,50,52,66}$

- Effect on EMS system performance measures ${ }^{66,67}$

- ED attendance or hospital admission after EMS episode ${ }^{14,59}$

- ED bed hours ${ }^{21}$

- Hospital admissions ${ }^{48,49}$

- Number contacts with health system ${ }^{31,43,66}$

- Patient satisfaction $16,17,25,26,49,51,57-60,66-68$

- Patient perception of care received $d^{51,53}$

- Annual EMS transport cost estimates ${ }^{21}$

- Economic analysis modelling ${ }^{25}$

- Incremental cost-effectiveness analysis ${ }^{41}$

- Patient care costs ${ }^{66,67}$

- Cost benefit analysis ${ }^{43}$

- Cost to system 60,66

- Economic evaluation ${ }^{47}$
- Consensus required on what a "successful" referral is, e.g., calls that are referred and referral agency does not return call back to 911. Reported as: number of calls "successfully" referred/total eligible calls. ${ }^{26}$

- May be defined as: number of EMS responses for all EMS patients, for all those eligible for response, or for those pre-enrolled in alternative program. ${ }^{21}$

- EMS calls are reviewed and judged if unnecessary by an expert panel. ${ }^{28}$ Multidisciplinary panel should be independent from study and members should have an understanding of EMS practice.

- May be defined as: how many previous EMS calls appear to meet criteria for alternative program. ${ }^{29}$

- A non-transport may be defined as paramedics not transporting the patient from scene. There could be various reasons.

- Consensus on which system performance measures are most important, e.g., emergency call response time.

- Between 0 - 28 days after EMS episode ${ }^{59}$

- Collect by follow-up patient interview. ${ }^{16,26,51,53}$

- Collect by follow-up patient interview. ${ }^{16,26,51,53}$

- Clearly report on what costs are included in analysis

- Categorize methodological approach if potential savings/cost ${ }^{42}$ or actual savings/cost ${ }^{60,66}$ 


\section{Table 4. (Continued)}

Outcome Type

(Structure, Process,

System, Outcome)

Outcome Category

Specific Outcome Options

Measurement Considerations

Process Accuracy of

Decision

- Accuracy of dispatch software to identify appropriate cases $^{23}$

- Safety of EMT decision to assign transport options $^{63}$

- Under/over-triage rate ${ }^{62,63}$

- Decision on medical necessity (if patient required $\mathrm{ED}$ care), triage decision ${ }^{1,14,18,44,54,62,63}$

- Accuracy of diagnosis ${ }^{46}$

- ALS interventions administered ${ }^{24}$

- Number of investigations ${ }^{43,60}$

- Paramedic compliance with protocols ${ }^{57,66}$

- Decision support algorithms used and followed ${ }^{66}$

- Patient compliance with treatment recommendations given ${ }^{31,53}$

- Paramedic documentation 45,66

- To identify target populations ${ }^{29,30,55}$

- How practitioners make decisions ${ }^{52}$

- Decision should be categorized and reported by provider type. ${ }^{12}$

- Paramedic documented decision compared to patient outcome ${ }^{62,64}$ or expert panel decision and reported with sensitivity and specificity ${ }^{62}$

- Paramedics answer the following questions for each patient (standard paramedic management delivered): "Could this patient have been safely transported by a non-medical transport service?" and "Could this patient have been safely transported to a clinic or urgent care center?"54

- Clinician decision may be compared to expert panel or physicians, ${ }^{1,3,13,27,50}$ or if patient seen in ED or admitted to hospital $^{12,18,44}$

- Agreement should be measured and reported with sensitivity and specificity. ${ }^{1,50}$

- Consensus definition of "medical necessity" and "appropriate decision" required, and should be clearly reported.

- Clinician diagnosis can be compared to "gold standard," such as diagnosis at 24 hours. ${ }^{46}$

- Characteristics of patients who paramedics judged were "medically unnecessary" to transport to ED described. ${ }^{55}$

- Analyze cases to determine key aspects of decision making styles. ${ }^{52}$ 
three randomized controlled trials, three studies with a non-randomized comparison group, and three studies from another population or simulation. There were four protocols of controlled trials. The outcome categories most reported were service utilization $(n=14)$, safety $(n=12)$, patient satisfaction $(n=10)$, accuracy of decision $(n=9)$, and clinical outcomes $(n=9)$ (Table 3). In total, there were 50 different unique outcomes reported. The outcome categories with the greatest number of specific different outcomes were service utilization $(n=8)$, accuracy of decision $(n=7)$, clinical outcomes $(n=7)$, call times $(n=7)$, process outcomes $(n=6)$, safety $(n=6)$, cost $(n=5)$, patient satisfaction $(n=2)$, and other $(n=2)$.

\section{DISCUSSION}

The purpose of this broad scoping literature review was to identify and catalogue the outcome measures used to study and report on alternatives to EMS. Scoping reviews are valuable for mapping complex topics to increase understanding. ${ }^{7}$ As we sought to identify all outcome measures used in any type of "alternative to traditional EMS dispatch or transport" program, this approach was most suitable. Forty-eight publications of over 1,000,000 patients for a wide variety of programs and interventions were located and included. These publications were very heterogeneous in design, population, and outcomes. The categories of outcomes most reported in the alternatives to traditional EMS dispatch reports were service utilization and accuracy of decisions, with 12 different sub-categories. In the alternatives to traditional EMS transport reports, the outcome categories with the most reported outcomes were service utilization, safety, clinical outcomes, and call times, with 29 sub-categories. This review revealed that similar outcomes are measured in many different ways. For example, adverse events have been examined by asking patients directly, through retrospective examination of health records, and through panel assessments of whether decisions were safe. ${ }^{13,16,25}$ This variance was also identified in a recent focus group study of US EMS services, in which safety was assessed in multiple ways, including by retrospective chart reviews and follow-up phone calls with patients. ${ }^{4}$

Just as this review was useful for identifying outcomes that are used frequently, it is also valuable to shed light on under-reported outcome categories. In the alternatives to dispatch studies, clinical outcomes were not reported, and process outcomes were only reported in one study. The least-used outcome category in the "alternatives to transport" studies was the "other" outcomes to identify a potential target program population, reported in two studies, ${ }^{29,30}$ and uptake of advice by patients was even more infrequent, studied in just one publication. ${ }^{31}$ There are additional outcomes that could be of great value but that have not been used in any of these studies. For example, it would be of great value to assess the effect of such services on the response times of other EMS units as a times outcome.

\section{Alternative to dispatch}

As noted in a recent systematic review, ambulancedispatch-based secondary triage has been implemented in many locations, as a strategy to avoid dispatching ambulances to low-priority calls, which may also help with ED overcrowding challenges. ${ }^{32}$ Our scoping review captured the same articles included in this review by Eastwood et al, with the exception of a descriptive publication that did not report on outcomes. ${ }^{33}$ Our review located 15 publications (16 studies), of which the majority reported on EMS dispatch programs that diverted callers to either nurse-advice lines or alternate EMS services (other than standard emergency ambulance dispatch). The remaining programs provided advice or self-care instructions, or identified low-acuity patients who did not require an EMS response. To fully understand the effect of all of these programs that are alternatives to EMS dispatch, uniform outcome measures need to be employed across studies. This review examined the structure, safety, and success of such systems, and found evidence from six studies that the services were safe and patients were satisfied. Success of referrals, an outcome of the review, was not welladdressed in the results. Recent studies with supportive results were of low quality and with small- to moderatesized samples. These studies focused on reporting outcomes on service utilization.

\section{Alternatives to transport}

Previous authors have noted that determining the need for EMS transport cannot be solely based on the medical necessity for the patient to be seen in the ED. ${ }^{34}$ As noted by Chu et al, any tools to evaluate eligibly of low-acuity patients for an alternative to ambulance transport must include assessing patient ability to 
ambulate. ${ }^{35}$ Some studies have explored the complexity of transport decisions and the many factors that must be considered by EMS responders. ${ }^{36,37}$ Many of the 33 studies included in this review include strategies within the programs reported to provide patients with access to other services, expanded care on scene, or other means of transport to the ED. The body of knowledge related to "mobile integrated healthcare" or "community paramedicine" has become multi-faceted and complex, with many services tailored specifically for the population or community they are aiming to better serve. This reinforces the need for consistent outcome measures for evidence-users to increase their understanding of the effects of such programs. In our review, recent supportive studies included a randomized controlled trial and a large comparison study, both of which reported on service utilization outcomes, and a cost analysis based on a large sample. ${ }^{42,52,59}$ There were four studies included that contained opposing evidence, all of which were studies without a comparison group. Also included were four protocol manuscripts, which describe upcoming randomized trials, which will add valuable high-quality evidence.

\section{Next steps}

Two key challenges have been identified that impede further evidence synthesis in this body of literature. 1) The programs or interventions studied are extremely heterogeneous. We appreciate the importance of developing programs that meet specific community needs. However, these programs or interventions should be grouped into main categories that will facilitate comparison and pooling of findings. For example, studies should first be categorized according to whether they were an alternative to EMS dispatch or transport. If it is an alternative to EMS dispatch, the intervention service may be categorized as: to nurse-advice line, to referral pathway (such as family practice clinic), to alternative EMS response, given advice or self-care instructions, or other. If it is an alternative to EMS transport, the intervention service may be categorized as: paramedic treat and release, EMS-initiated nontransport, call or refer to another health service or referral pathway, consult with a physician or other provider, expanded scope EMS delivery, alternative mode of transport to the ED, or other. 2) Many different approaches have been used to measure similar outcomes. What is now required is to carry out a consensus project to determine which outcomes are most important to use. A taxonomy of standard terms as well as outcome definitions would allow valid comparisons across systems. In the US, a consensus session led to the publication of a National Agenda for Community Paramedicine Research, ${ }^{38}$ which determined research priorities, but did not give direction on which are the ideal outcomes to use, and what method(s) to use. Categorizing outcomes into type by process (measures of actions or functions), system (measures of how the system works), and outcome (patient-related changes in outcome that are attributable to care received) would further harmonize research comparisons (Table 4).

\section{LIMITATIONS}

A scoping review was determined to be an appropriate evidence synthesis strategy for this topic, as opposed to a structured systematic review, as the topic was multifaceted, the question could not be narrowly defined, and it was important to map all studies conducted and include all levels of evidence, all of which are key strengths of the scoping review approach. ${ }^{7}$ There are many different types of alternative EMS programs included in this body of literature, which spans over two decades, during which time EMS has changed significantly, all of which may affect the suitability of the outcome measures collected. These alternative EMS programs have often developed out of local needs in attempts to better serve their patient population with the resources that are available. ${ }^{32}$ While appropriate, this leads to difficulty in understanding what the findings mean in aggregate, and certainly prohibits quantitative pooling in a systematic review. This heterogeneity can also significantly limit the generalizability of the findings to contemporary EMS systems; however, the outcomes used can still be considered for use in modern research and quality projects. Some publications provided limited information on their EMS settings or programs.

\section{CONCLUSION}

In this broad scoping review on alternatives to traditional EMS dispatch and transport, numerous outcome measures used to measure and report on these interventions and programs were identified and catalogued. Researchers and program leaders should achieve consensus on the most important outcome measures to be 
used in future research studies, program evaluations and quality assessments of these programs.

Acknowledgements: Thank you to Peter Rose, ACP, who assisted with the grey literature search, and to Fahd Al-Dhalaan, Dalhousie University medical student, who assisted with data abstraction.

Competing interests: The authors would like to declare funding information: Emergency Health Services provided funding for the MLIS candidate to design and conduct bibliographic searches. EHS Operations Management provided in-kind support.

\section{SUPPLEMENTARY MATERIAL}

To view supplementary material for this article, please visit http://dx.doi.org/10.1017/cem.2014.59

\section{REFERENCES}

1. Gratton MC, Ellison SR, Hunt J, et al. Prospective determination of medical necessity for ambulance transport by paramedics. Prehosp Emerg Care 2003;7(4):466-9.

2. Neely KW. Ambulance transports: What are the alternatives? Acad Emerg Med 1997;4(12):1103-6.

3. Farand L, Leprohon J, Kalina M, et al. The role of protocols and professional judgement in emergency medical dispatching. Eur 7 Emerg Med 1995;2(3):136-48.

4. Morganti KG, Alpert A, Margolis G, et al. The state of innovative emergency medical service programs in the united states. Prehosp Emerg Care 2014;18(1):76-85.

5. Snooks HA, Dale J, Hartley-Sharpe C, et al. On-scene alternatives for emergency ambulance crews attending patients who do not need to travel to the accident and emergency department: A review of the literature. Emerg Med $\mathcal{F} 2004 ; 21(2): 212-5$.

6. Bigham BL, Kennedy SM, Drennan I, et al. Expanding paramedic scope of practice in the community: A systematic review of the literature. Prehosp Emerg Care 2013;17(3): 361-72.

7. Arksey H, O'Malley L. Scoping studies: Towards a methodological framework. Int 7 Soc Res Meth 2005;8(1):19-32.

8. Schlosser RW, Wendt O, Bhavnani S, et al. Use of information-seeking strategies for developing systematic reviews and engaging in evidence-based practice: The application of traditional and comprehensive pearl growing. A review. Int 7 Lang Commun Disord 2006;41(5):567-82.

9. Booth A. Unpacking your literature search toolbox: On search styles and tactics. Health Info Libr 7 2008;25(4):313-7.

10. McGowan J, Sampson M. Systematic reviews need systematic searchers. $\mathcal{f}$ Med Libr Assoc 2005;93(1):74-80.

11. Papaioannou D, Sutton A, Carroll C, et al. Literature searching for social science systematic reviews: Consideration of a range of search techniques. Health Info Libr $\mathcal{7}$ 2010;27(2):114-22.

12. Dale J, Higgins J, Williams S, et al. Computer assisted assessment and advice for "non-serious" 999 ambulance service callers: The potential impact on ambulance despatch. Emerg Med 7 2003;20(2):178-83.

13. Dale J, Williams S, Foster T, et al. Safety of telephone consultation for "non-serious" emergency ambulance service patients. Qual Saf Health Care 2004;13(5):363-73.

14. Schaefer RA, Rea TD, Plorde M, et al. An emergency medical services program of alternate destination of patient care. Prehosp Emerg Care 2002;6(3):309-14.

15. Schmidt T, Neely KW, Adams AL, et al. Is it possible to safely triage callers to EMS dispatch centers to alternative resources? Prehosp Emerg Care 2003;7(3):368-74.

16. Smith WR, Culley L, Plorde M, et al. Emergency medical services telephone referral program: An alternative approach to nonurgent 911 calls. Prehosp Emerg Care 2001;5(2): 174-80.

17. Snooks H, Kearsley N, Dale J. Towards primary care for non-serious 999 callers: Results of a controlled study of "treat and refer" protocols for ambulance crews. Qual Saf Health Care 2004;13(6):435-43.

18. Studnek JR, Thestrup L, Blackwell T, et al. Utilization of prehospital dispatch protocols to identify low-acuity patients. Prehosp Emerg Care 2012;16(2):204-9.

19. The Canadian Agency for Drugs and Technologies in Health. Grey matters: A practical search tool for evidence-based medicine. 2014. Available at: http://www.cadth.ca/en/resources/ finding-evidence-is/grey-matters (accessed December 4, 2012).

20. Giustini D. Finding the hard to finds: Searching for grey literature. 2012. Available at: http://blog.openmedicine.ca/ node/253 (accessed December 4, 2012).

21. AHRQ healthcare innovations exchange. Trained paramedics provide ongoing support to frequent 911 callers, reducing use of ambulance and emergency department services. 2012. Available at: http://innovations.ahrq.gov/ content.aspx?id=3343 (accessed December 4, 2012).

22. Crowther L, Williams R. Nurse interventions in ambulance command-and-control centres. Emerg Nurse 2009;17(8): 22-25.

23. Gray JT, Walker A. AMPDS categories: Are they an appropriate method to select cases for extended role ambulance practitioners? Emerg Med 7 2008;25(9):601-3.

24. Key CB, Pepe PE, Persse DE, et al. Can first responders be sent to selected 9-1-1 emergency medical services calls without an ambulance? Acad Emerg Med 2003;10(4): 339-46.

25. Turner J, Snooks H, Youren A, et al. The costs and benefits of managing some low priority 999 ambulance calls by NHS direct nurse advisers. Sheffield, UK: The University of Sheffield; 2006. Available at: http://www.nets.nihr.ac.uk/_data/assets/ pdf_file/0011/64469/FR-08-1304-43.pdf (accessed December 4, 2012).

26. Infinger A, Studnek JR, Hawkins E, et al. Implementation of prehospital dispatch protocols that triage low-acuity patients to advice-line nurses. Prehosp Emerg Care 2013;17(4): 481-5.

27. Leprohon J, Patel VL. Decision-making strategies for telephone triage in emergency medical services. Med Decis Making 1995;15(3):240-53.

28. Alizadeh R, Panahi F, Saghafinia M, et al. Impact of trauma dispatch algorithm software on the rate of missions of 
emergency medical services. Trauma Mon 2012;17(3): 319-22.

29. Haskins PA, Ellis DG, Mayrose J. Predicted utilization of emergency medical services telemedicine in decreasing ambulance transports. Prehosp Emerg Care 2002;6(4):445-8.

30. Kamper M, Mahoney BD, Nelson S, et al. Feasibility of paramedic treatment and referral of minor illnesses and injuries. Prebosp Emerg Care 2001;5(4):371-8.

31. Mikolaizak AS, Simpson PM, Tiedemann A, et al. Intervention to prevent further falls in older people who call an ambulance as a result of a fall: A protocol for the iPREFER randomised controlled trial. BMC Health Serv Res 2013;13:360, doi:10.1186/1472-6963-13-360.

32. Eastwood K, Morgans A, Smith K, et al. Secondary triage in prehospital emergency ambulance services: A systematic review. Emerg Med 7 2014, e-pub, doi:10.1136/emermed2013-203120.

33. Fox C, Rodriguez C, McSwain NE. EMT telephone triage. EMT 7 1981;5(6):410-5.

34. Brown LH, Hubble MW, Cone DC, et al. Paramedic determinations of medical necessity: A meta-analysis. Prebosp Emerg Care 2009;13(4):516-27.

35. Chu KH, Gregor MA, Maio RF, et al. Derivation and validation of criteria for determining the appropriateness of nonemergency ambulance transports. Prehosp Emerg Care 1997;1(4):219-26.

36. Porter A, Snooks H, Youren A, et al. 'Should I stay or should I go?' deciding whether to go to hospital after a 999 call. $\mathcal{f}$ Health Serv Res Policy 2007;12(S1):S1-32-8.

37. Jensen JL, Travers AH, Marshall EG, et al. Insights into the implementation and operation of a novel paramedic long-term care program. Prehosp Emerg Care 2014;18(1): 86-91.

38. Patterson DG, Skillman SM. A national agenda for community paramedicine research. Seattle, WA: WWAMI Rural Health Research Centre, University of Washington; 2013. Available from: http://depts.washington.edu/uwrhrc/ uploads/CP_Agenda.pdf (accessed December 5, 2012).

39. Sayre MR, O'Connor RE, Atkins DL, et al. Part 2: Evidence evaluation and management of potential or perceived conflicts of interest: 2010 American Heart Association guidelines for cardiopulmonary resuscitation and emergency cardiovascular care. Circulation 2012;122(18S3):S657-64.

40. Dalhousie University Division of EMS (2014). Canadian Prehospital Evidence-based Practice Project. Level and Direction of Evidence. Available from: https://emspep.cdha. nshealth.ca/MethodLOEChart.aspx (accessed January 10, 2013).

41. Widiatmoko D, Machen I, Dickinson A, et al. Developing a new response to non-urgent emergency calls: Evaluation of a nurse and paramedic partnership intervention. Prim Health Care Res Dev 2008;9(3):183.

42. Alpert A, Morganti KG, Margolis GS, et al. Giving EMS flexibility in transporting low-acuity patients could generate substantial Medicare savings. Health Aff (Millwood) 2013;32 (12):2142-8.

43. Arendts G, Sim M, Johnston S, et al. ParaMED home: A protocol for a randomised controlled trial of paramedic assessment and referral to access medical care at home. $B M C$ Emerg Med 2011;11:7.
44. Coates D, Rawstorne S, Benger J. Can emergency care practitioners differentiate between an avoided emergency department attendance and an avoided admission? Emerg Med 7 2012;29(10):838-41.

45. Cooper S, Barrett B, Black S, et al. The emerging role of the emergency care practitioner. Emerg Med 7 2004;21(5): 614-8.

46. Cooper S, O'Carroll J, Jenkin A, et al. Emergency care practitioners (ECP): Practice and performance in the UK west country-a case study. Int Emerg Nurs 2008;16(3): 180-4.

47. Dixon S, Mason S, Knowles E, et al. Is it cost effective to introduce paramedic practitioners for older people to the ambulance service? results of a cluster randomised controlled trial. Emerg Med 7 2009;26(6):446-51.

48. Gray JT, Walker A. Avoiding admissions from the ambulance service: A review of elderly patients with falls and patients with breathing difficulties seen by emergency care practitioners in South Yorkshire. Emerg Med 7 2008;25(3):168-71.

49. Haines CJ, Lutes RE, Blaser M, et al. Paramedic initiated non-transport of pediatric patients. Prehosp Emerg Care 2006;10(2):213-9.

50. Halter M, Close JCT, Snooks H, et al. Fit to be left: Can ambulance staff use an assessment tool to decide if an older person who bas fallen can be safely left at home?. London, UK: Department of Health Research Programme to Support Implementation of the National Service Framework for Older People; 2005. Available from: http://www.healthcare. ac.uk/research/documents/fit-to-be-left-report.pdf (accessed March 15, 2013).

51. Halter M, Marlow T, Tye C, et al. Patients' experiences of care provided by emergency care practitioners and traditional ambulance practitioners: A survey from the London ambulance service. Emerg Med 7 2006;23(11):865-6.

52. Halter M, Ellison G. Evaluation of the emergency care practitioner in London: A study of the processes and outcomes of clinical decision making. London, UK: Faculty of Health and Social Care Sciences, Kingston University, St. George's, University of London; 2008.

53. Halter M, Marlow T, Mohammed D, et al. A patient survey of out-of-hours care provided by emergency care practitioners. BMC Emerg Med 2007;7:4, doi:10.1186/1471227X-7-4.

54. Hauswald M. Can paramedics safely decide which patients do not need ambulance transport or emergency department care? Prehosp Emerg Care 2002;6(4):383-6.

55. Hjalte L, Suserud BO, Herlitz J, et al. Why are people without medical needs transported by ambulance? A study of indications for pre-hospital care. Eur 7 Emerg Med 2007; 14(3):151-6.

56. Knapp BJ, Tsuchitani SN, Sheele JM, et al. Prospective evaluation of an emergency medical services-administered alternative transport protocol. Prehosp Emerg Care 2009; 13(4):432-6.

57. Lerner EB, Billittier AJ, Lance DR, et al. Can paramedics safely treat and discharge hypoglycemic patients in the field? Am 7 Emerg Med 2003;21(2):115-20.

58. Jensen JL, Travers AH, Bardua DJ, et al. Transport outcomes and dispatch determinants in a paramedic long-term care program: A pilot study. CFEM 2013;15(4):206-13. 
59. Mason S, Knowles E, Colwell B, et al. Effectiveness of paramedic practitioners in attending 999 calls from elderly people in the community: Cluster randomised controlled trial. BM7 2007;335(7626):919.

60. Mason S, O'Keeffe C, Coleman P, et al. Effectiveness of emergency care practitioners working within existing emergency service models of care. Emerg Med 7 2007;24(4): 239-43.

61. Mason S, Knowles E, Freeman J, et al. Safety of paramedics with extended skills. Acad Emerg Med 2008;15(7):607-12.

62. Pointer JE, Levitt MA, Young JC, et al. Can paramedics using guidelines accurately triage patients? Ann Emerg Med 2001;38(3):268-77.

63. Schmidt TA, Atcheson R, Federiuk C, et al. Hospital follow-up of patients categorized as not needing an ambulance using a set of emergency medical technician protocols. Prehosp Emerg Care 2001;5(4):366-70.

64. Silvestri S, Rothrock SG, Kennedy D, et al. Can paramedics accurately identify patients who do not require emergency department care? Prehosp Emerg Care 2002;6(4):387-90.
65. Snooks H, Foster T, Nicholl J. Results of an evaluation of the effectiveness of triage and direct transportation to minor injuries units by ambulance crews. Emerg Med 7 2004;21(1): 105-11.

66. Snooks H, Cheung WY, Close J, et al. Support and assessment for fall emergency referrals (SAFER 1) trial protocol. Computerised on-scene decision support for emergency ambulance staff to assess and plan care for older people who have fallen: Evaluation of costs and benefits using a pragmatic cluster randomised trial. BMC Emerg Med 2010;10:2.

67. Snooks H, Anthony R, Chatters R, et al. Support and assessment for fall emergency referrals (SAFER 2) research protocol: Cluster randomised trial of the clinical and cost effectiveness of new protocols for emergency ambulance paramedics to assess and refer to appropriate communitybased care. BM7 Open 2012;2(6):e002169, doi:10.1136/ bmjopen.2012-002169.

68. Zachariah BS, Bryan D, Pepe PE, et al. Follow-up and outcome of patients who decline or are denied transport by EMS. Prehosp Disaster Med 1992;7(4):359-63. 